\title{
Rayleigh's quotient-based damage detection algorithm: Theoretical concepts, computational techniques, and field implementation strategies
}

\section{NJOMO WANDJI, Wilfried}

Published in:

Structural Health Monitoring

Link to article, DOI:

$10.1177 / 1475921717691018$

Publication date:

2018

Document Version

Peer reviewed version

Link back to DTU Orbit

Citation (APA):

NJOMO WANDJI, W. (2018). Rayleigh's quotient-based damage detection algorithm: Theoretical concepts, computational techniques, and field implementation strategies. Structural Health Monitoring, 17(2), 285-297. https://doi.org/10.1177/1475921717691018

\section{General rights}

Copyright and moral rights for the publications made accessible in the public portal are retained by the authors and/or other copyright owners and it is a condition of accessing publications that users recognise and abide by the legal requirements associated with these rights.

- Users may download and print one copy of any publication from the public portal for the purpose of private study or research.

- You may not further distribute the material or use it for any profit-making activity or commercial gain

- You may freely distribute the URL identifying the publication in the public portal 


\title{
Rayleigh's quotient-based damage detection algorithm: Theoretical concepts, computational techniques, and field implementation strategies
}

Structural Health Monitoring $1-13$

(C) The Author(s) 2017

Reprints and permissions: sagepub.co.uk/journalsPermissions.nav DOI: $10.1177 / 1475921717691018$ journals.sagepub.com/home/shm @SAGE

\author{
Wilfried Njomo Wandji
}

\begin{abstract}
This article proposes a Rayleigh's quotient-based damage detection algorithm. It aims at efficiently revealing nascent structural changes on a given structure with the capability to differentiate between an actual damage and a change in operational conditions. The first three damage detection levels are targeted: existence, location, and severity. The proposed algorithm is analytically developed from the dynamics theory and the virtual energy principle. Some computational techniques are proposed for carrying out computations, including discretization, integration, derivation, and suitable optimization methods. Field implementation strategies are also considered for the purpose of online damage monitoring. In order to prove the efficiency of this strategy, one experimental and three numerical case studies were conducted. The proposed algorithm successfully detected the damage in all simulated cases and estimated the damage severity with acceptable accuracy. The conclusion is that the proposed algorithm was able to efficiently detect damage appearance in a range of structures for various damage levels and locations, and under different operational conditions.
\end{abstract}

\section{Keywords}

Damage detection algorithm, operational monitoring, Rayleigh's quotient, principle of virtual displacement, damping, optimization, generalized fractal dimension

\section{Introduction}

Structural failures can cause significant losses in many societal aspects: economy, ecology, infrastructure, and human lives. In some cases, failures are the ultimate outcome of a progressing damage that could have been stopped if detected earlier. Different concepts have been used with the purpose of detecting damage in structures; they include among others vibration, strain, acoustic emission, image, and laser-based monitoring.

Vibration-based methods are one of the oldest methods as they began to be utilized during the late 1970s and continue to attract some researchers. ${ }^{1}$ In the trail of this method, identifying damage can be derived either from shape, slope, curvature, modal assurance criterion (MAC) and its derivatives, soft computing-based methods, gapped smoothing method (GSM), fractal dimension (FD), or from statistical techniques. Each of these has been investigated by various authors.

For example, Abdo and Hori $^{2}$ proposed a method based on modal slope to detect damage. On a numerical model of a plate, damages are simulated by freeing relevant nodes after mesh generation. A similar damage simulation strategy has also been adopted by Yan et al., ${ }^{3}$ who assumed damage as a reduction of the local stiffness in a numerical model of a composite vessel. Based on modal and sensor norms, Gawronski and Sawicki ${ }^{4}$ attempted with good success to detect damage on a truss in laboratory conditions. In addition, Elshafey et al. ${ }^{5}$ discussed the damage detection in offshore jacket platforms subjected to random loads using a combined method of random decrement signature and neural network. Although this work considered changes in support condition, the study ignored changes in the deck mass. Moreover, this method relies on the intact state of the structure, which is not easily recoverable, especially for existing structures.

Saheli et al. ${ }^{6}$ presented a technique of damage detection based on the real and imaginary parts of measured

DTU Wind Energy, Technical University of Denmark, Roskilde, Denmark

\section{Corresponding author:}

Wilfried Njomo Wandji, DTU Wind Energy, Technical University of Denmark, Frederiksborgvej 399, 4000 Roskilde, Denmark.

Email:wilw@dtu.dk 
frequency response functions (FRF). By comparing intact and damaged structures, the authors observed that an abnormality is found in residual FRF shapes in damaged sites which are detected via GSM. To determine this, a numerical ruler of $900 \mathrm{~mm} \times 6.5 \mathrm{~mm}$ $\times 25.5 \mathrm{~mm}$ and an experimental one of $800 \mathrm{~mm} \times 25$ $\mathrm{mm} \times 50 \mathrm{~mm}$ are considered. Yoon et al. ${ }^{7}$ presented a procedure for locating variability in structural stiffness. The one-dimensional (1D) GSM is extended to twodimensional (2D) GSM. Similarly, wavelet method continues to attract scientific interest, either using a baseline structure ${ }^{8}$ or being reference-free. ${ }^{9}$ Wang and Qiao $^{10}$ developed the generalized fractal dimension (GFD) from the conventional definition of FD. They observed that GFD shows similar features as spatial wavelet transformation, but outperforms it due to several reasons: (1) simpler in calculation, (2) less storage space needed for data, (3) easier to determine damage severity, and (4) ideal for online data processing as only a small segment of the measured signal is required. These researchers used abrupt changes of shape or curvature at a given structural point compared to its vicinity to detect damages. These techniques do not need any baseline structure. However, GSM results interpretation may be difficult as the algorithm only detects damaged edges. Moreover, the sizing index used in their study was only indicative and does not have any physical meaning.

Viero and Roitman ${ }^{11}$ reported on the evaluation of the performance of MAC, coordinate modal assurance criterion (COMAC), modal scale factor (MSF), mode shape relative difference method (RD), and change in a modal vector perpendicular to the predominant modal direction as damage identification methods applied on two different small-scale hydroelastic models of fixed offshore platforms. It is shown that MAC and MSF are sensitive to both damages and deck mass changes. The influence of damages and deck mass changes can be distinguished by analyzing the results from COMAC, RD, and the modal vector. Ruotolo et al. ${ }^{12}$ compared results of the application to simulated data of a typical offshore structure using two recently proposed methods: a novelty detection method and a singular value decomposition (SVD)-based technique. Five different operational conditions and four different damage levels are imposed to the structure. It has been shown that these two methods were generally sensitive to each case. However, in some cases, the investigators were not able to recognize damage. Furthermore, the SVD-based technique requires data related to the undamaged structure, which are not always available.

Recently, non-deterministic damage detection techniques have emerged. The main strategy is based on statistical methods. Nguyen et al. ${ }^{13}$ investigated the effects and uncertainties of wireless sensor network on output-only modal analysis employing merged data of multiple tests using a lab bridge model. A full size bridge has been utilized as case study by Reynders et al. $^{14}$ to present the effectiveness of a nonlinear output-only method based on kernel principal component analysis. Various other multivariate statistical techniques have been employed in continuous damage detection campaigns on historical structures ${ }^{15}$ and bridges. ${ }^{16}$ They showed that robust output-only modal properties can be efficient in discriminating actual damage signature from environmental and operational influences.

Each individual technique presented above shows a combination of good features and drawbacks. This suggests superimposing these techniques and retaining the desirable features from each of them. In order to combine the features of many of these strategies together into a whole algorithm, a procedure based on Rayleigh's quotient has been developed. It encompasses the properties of methods based on modal shape, modal slope, modal curvature, frequency shift, and GSM or FD to distinguish efficiently between the effects of damage occurrence and the effects of mass variation on the vibration signature of a given structure. It fulfills the first three levels of damage detection: ${ }^{1}$ existence, localization, and severity. The severity resulting from this procedure is not an abstract index, but a geometric measure of the damage.

In the following sections, basic vibrational theory is recalled. From that, the proposed algorithm is derived and computational techniques are proposed. A workflow scheme is drawn and is applied on three numerical case studies and one additional experimental case. Results and discussions are presented, and possibilities to further research are outlined.

\section{Vibrational theory}

Under vibration, structure motions are described by the differential equation ${ }^{17,18}$

$$
\underline{\underline{M}} \underline{\ddot{u}(t)}+\underline{\underline{C}} \underline{u}(t)+\underline{\underline{K}} \underline{u(t)}=\underline{P(t)}
$$

where $\underline{\underline{M}}, \underline{C}$, and $\underline{\underline{K}}$ are, respectively, the mass, damping, and stiffness matrices of the discretized model and $P(t)$ is the disturbance that initiates the vibration, or the action that sustains the motions.

The undamped modal frequencies, $w_{u}$, and the modal shapes, $\varnothing$, can be obtained as, respectively, the eigenvalues and the eigenvectors of the system $(\underline{K}, \underline{\underline{M}})$.

Assuming that the damping matrix can be expressed as a linear combination of the mass and stiffness matrices such as defined by Rayleigh: ${ }^{12} \underline{\underline{C}}=\alpha \underline{\underline{K}}+\beta \underline{\underline{M}}$, the damped modal frequency can be obtained for each 


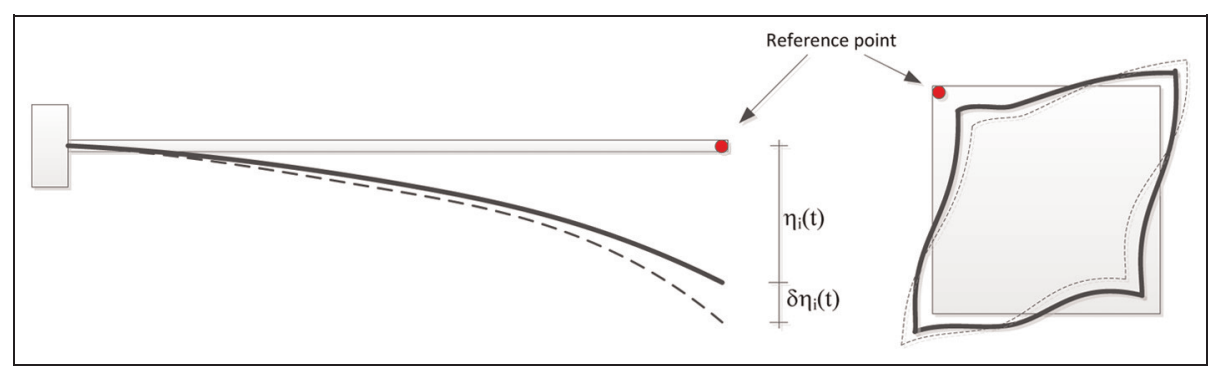

Figure I. Generalized coordinates' picture.

mode $i$ from $w_{d, i}=w_{u, i} \sqrt{1-\zeta_{i}^{2}},{ }^{17} \zeta_{i}$ is the modal damping ratio, $\zeta_{i}=\beta /\left(2 w_{u, i}\right)+\alpha w_{u, i} / 2$.

Besides, the undamped modal frequency for mode $i$ can be expressed as $w_{u, i}=\sqrt{k_{i} / m_{i}}$, with $m_{i}=\underline{\varnothing_{i}^{T}} \underline{\underline{M}} \underline{\varnothing_{i}}$, $k_{i}=\varnothing_{i}^{T} \underline{\underline{K}} \underline{\varnothing_{i}}$. Therefore, the Rayleigh's quotient can be stated as

$$
\frac{w_{d, i}}{\sqrt{1-\zeta_{i}^{2}}}=\sqrt{\frac{k_{i}}{m_{i}}} \text { or } \frac{w_{d, i}^{2}}{1-\zeta_{i}^{2}}=\frac{k_{i}}{m_{i}}
$$

\section{Typology of damping}

Damping can be caused by various phenomena. Karamchandani et al. ${ }^{19}$ have distinguished the following: (1) aerodynamic damping proportional to the second (or higher) power of velocity, (2) structural (hysteretic) damping caused by molecular interaction in the material, (3) Coulomb's damping generated by the friction between surfaces, and (4) viscous damping due to contact with surrounding fluid. In a damped motion, all of these damping types can be present and act concurrently. In structural engineering, it is a cumbersome task to evaluate the individual contribution of each of them. In common cases, however, this is not required. In general, the total damping effect is measured in terms of viscous damping. Under this assumption, the damping effect will be related to the velocity which is easily quantifiable. In the rest of this article, damping will be evaluated in terms of viscous damping, but kept at its actual structural location (either at the body level, at its supports, or on its surfaces in contact with the ambient fluid.)

\section{Generalized coordinates}

In many practical cases, the modal shapes can be represented by a spatial surface or a spatial line, $\varphi_{i}(x, y, z)$, that satisfies the boundary conditions. Different possibilities are conceivable; but the best modal shape is the one minimizing the potential energy of the system. If a point of coordinates $\left(x_{0}, y_{0}, z_{0}\right)$ is selected on the structure as a reference point, $\boldsymbol{\varphi}_{i}$ can be scaled such that $\boldsymbol{\varphi}_{i}\left(x_{0}, y_{0}, z_{0}\right)_{\text {scaled }}=\varphi_{i}\left(x_{0}, y_{0}, z_{0}\right)=1$. Now, it is sufficient to monitor just the modal motion of the said reference point, $\eta_{i}(t)$, because the modal motion of other points can be deduced by $H_{i}(x, y, z, t)=\varphi_{i}(x, y, z) \eta_{i}(t)$, where $\varphi_{i}$ is the scaled version of $\boldsymbol{\varphi}_{i}$. Figure 1 depicts the generalized coordinates' reduction. In this figure, given that the thick black line has the known shape, its entire locus can be completely determined by only tracking $\eta_{\mathrm{i}}(t)$.

\section{Derivations for the proposed algorithm}

For most civil engineering structures, global translational mode, global bending mode, global torsional mode, and global shearing mode can clearly be distinguished. Whichever global mode is observed, the principle of virtual displacement can be applied. It states that, due to a virtual displacement $\delta H$, the external and internal virtual works are the same: $\delta W_{\text {ext }}=\delta W_{\text {int }}$. Each of these internal and external virtual works needs to be evaluated. In Figure 1, assuming a virtual displacement at the reference point, these virtual works can be decomposed into individual effects of the mass, disturbance or load, damping, and stiffness. Hence, for mode $i$

$$
\begin{gathered}
\delta W_{i n t, i}=\delta W_{k, i} \\
\delta W_{e x t, i}=-\delta W_{m, i}-\delta W_{c, i}+\delta W_{p, i}
\end{gathered}
$$

Equating equations (3a) and (3b), the result for mode $i$ is $\delta W_{m, i}+\delta W_{c, i}+\delta W_{k, i}=\delta W_{p, i}$.

\section{Mass effect}

Mass causes inertial force whose virtual work is

$$
\begin{aligned}
\delta W_{m, i} & =\left[\ddot{H}_{i}(x, y, z, t) r(x, y, z) \rho(x, y, z) \delta H_{i}(x, y, z, t)\right] d V \\
& =\ddot{\eta}_{i}(t) \delta \eta_{i}(t) \oiiint \varphi_{i}^{2}(x, y, z) r(x, y, z) \rho(x, y, z) d x d y d z
\end{aligned}
$$


with $\rho(x, y, z)$ being the spatial field of mass density, and $r(x, y, z)$ is either equal to 1 in case of translation or equal to the distance separating the element $(x, y, z)$ to the axis in case of rotation.

\section{Disturbance or load effect}

Here, only the effects of loads acting on the structural domain surface, including nodal forces or moments, are considered. Body forces such as acceleration-induced loads can be readily combined with the mass effect discussed above. Others such as electromagnetic forces are rare in common civil engineering structures. Yet, they can still be implemented along this scheme. Therefore, the related disturbance virtual work is

$$
\begin{aligned}
\delta W_{p, i} & =\oiint\left[p_{i}(x, y, z, t) \delta H_{i}(x, y, z, t)\right] d S \\
& =\delta \eta_{i}(t) \oiint p_{i}(x, y, z, t) \varphi_{i}(x, y, z) d S
\end{aligned}
$$

\section{Damping effect}

The virtual work due to damping can be estimated by

$$
\begin{aligned}
\delta W_{c, i} & =\oiiint\left[\dot{H}_{i}(x, y, z, t) c_{i}(x, y, z) \delta H_{i}(x, y, z, t)\right] d V \\
& =\dot{\eta}_{i}(t) \delta \eta_{i}(t) \oiiint \varphi_{i}^{2}(x, y, z) c_{i}(x, y, z) d x d y d z
\end{aligned}
$$

with $c_{i}(x, y, z)$ being the spatial modal damping density field.

\section{Stiffness effect}

The stiffness-related virtual work is associated with strain energy. Here, four cases should be distinguished: translational, bending, shear, and torsional modes. The corresponding strain energies are evaluated considering that the structure remains elastic during vibrations. In case of heterogeneity, calculations should be done for each structural part where material moduli are constant.

For translation due to force $F_{x, i}$ along the $x$-axis and for mode $i$, applied on an element located at $(x, y, z)$ whose area perpendicularly to the $x$-axis is $A_{x}$, it can be written

$$
\begin{aligned}
\delta W_{k, i, x}^{t r} & =\oiiint\left[F_{x, i}(x, y, z, t) \delta \frac{\partial H_{i}(x, y, z, t)}{\partial x}\right] d V \\
& =\eta_{i}(t) \delta \eta_{i}(t) \oiiint\left\{E A_{x}(x, y, z)\left[\frac{\partial \varphi_{i}(x, y, z)}{\partial x}\right]^{2}\right\} d V
\end{aligned}
$$

To account for multi-axis translational deformation, the total energy is

$$
\begin{aligned}
& \delta W_{k, i}^{t r}=\eta_{i}(t) \delta \eta_{i}(t) \\
& \left.\oiiint_{E}\left\{\begin{array}{l}
A_{x}(x, y, z)\left[\frac{\partial \varphi_{i}(x, y, z)}{\partial x}\right]^{2}+A_{y}(x, y, z)\left[\frac{\partial \varphi_{i}(x, y, z)}{\partial y}\right]^{2} \\
+A_{z}(x, y, z)\left[\frac{\partial \varphi_{i}(x, y, z)}{\partial z}\right]^{2}
\end{array}\right]\right\} d x d y d z
\end{aligned}
$$

For bending mode due to moment, $M_{x, i}$, about $x$-axis and for mode $i$

$$
\begin{aligned}
\delta W_{k, i, x}^{m b} & \oiiint\left[M_{x, i}(x, y, z, t) \delta \frac{\partial^{2} H_{i}(x, y, z, t)}{\partial x^{2}}\right] d V \\
& =\eta_{i}(t) \delta \eta_{i}(t) \oiiint\left\{E I_{x}(x, y, z)\left[\frac{\partial^{2} \varphi_{i}(x, y, z)}{\partial x^{2}}\right]^{2}\right\} d V
\end{aligned}
$$

To account for multi-axis bending deformation, the total energy is

$\delta W_{k, i}^{m b}=\eta_{i}(t) \delta \eta_{i}(t)$
$\oiiint_{E}\left\{\left[\begin{array}{l}I_{x}(x, y, z)\left[\frac{\partial^{2} \varphi_{i}(x, y, z)}{\partial x^{2}}\right]^{2}+I_{y}(x, y, z)\left[\frac{\partial^{2} \varphi_{i}(x, y, z)}{\partial y^{2}}\right]^{2} \\ +I_{z}(x, y, z)\left[\frac{\partial^{2} \varphi_{i}(x, y, z)}{\partial z^{2}}\right]^{2}\end{array}\right]\right\} d x d y d z$

For torsional mode due to torque, $T_{x, i}$, about $x$-axis and for mode $i$

$$
\begin{aligned}
\delta W_{k, i, x}^{m t} & =\int\left[T_{x, i}(x, y, z, t) \delta \frac{\partial H_{i}(x, y, z, t)}{\partial x}\right] d V \\
& =\eta_{i}(t) \delta \eta_{i}(t) \oiiint\left\{G J_{x}(x, y, z)\left[\frac{\partial \varphi_{i}(x, y, z)}{\partial x}\right]^{2}\right\} d V
\end{aligned}
$$

To account for multi-axis torsional deformation, the total energy is

$$
\begin{aligned}
& \delta W_{k, i}^{m t}=\eta_{i}(t) \delta \eta_{i}(t) \\
& \left.\mathscr{H}_{E}\left\{\begin{array}{l}
J_{x}(x, y, z)\left[\frac{\partial \varphi_{i}(x, y, z)}{\partial x}\right]^{2}+J_{y}(x, y, z)\left[\frac{\partial \varphi_{i}(x, y, z)}{\partial y}\right]^{2} \\
+J_{z}(x, y, z)\left[\frac{\partial \varphi_{i}(x, y, z)}{\partial z}\right]^{2}
\end{array}\right]\right\} d x d y d z
\end{aligned}
$$

For shearing along $x$-axis applied on an effective shear area $\alpha A_{x}$

$$
\begin{aligned}
\delta W_{k, i, x}^{s r} & =\oiiint\left[Q_{x, i}(x, y, z, t) \delta \frac{\partial H_{i}(x, y, z, t)}{\partial x}\right] d V \\
& =\eta_{i}(t) \delta \eta_{i}(t) \oiiint\left\{G \alpha A_{x}(x, y, z)\left[\frac{\partial \varphi_{i}(x, y, z)}{\partial x}\right]^{2}\right\} d V
\end{aligned}
$$


To account for multi-axis shearing deformation, the total energy is

$$
\begin{aligned}
& \delta W_{k, i}^{s r}=\eta_{i}(t) \delta \eta_{i}(t) \\
& \mathscr{H}\left\{\left[\begin{array}{l}
\alpha A_{x}(x, y, z)\left[\frac{\partial \varphi_{i}(x, y, z)}{\partial x}\right]^{2}+\alpha A_{y}(x, y, z)\left[\frac{\partial \varphi_{i}(x, y, z)}{\partial y}\right]^{2} \\
+\alpha A_{z}(x, y, z)\left[\frac{\partial \varphi_{i}(x, y, z)}{\partial z}\right]^{2}
\end{array}\right]\right\} d x d y d z
\end{aligned}
$$

It is noteworthy to clarify that these virtual works are assumed to be exclusive while considering only a given global mode. In other words, one should consider only the stiffness work due to one deformation mode, say bending if the global vibration mode is bending. However, in some cases, the overall vibration mode of a whole structure can be different from the individual vibration modes of its elements. For example, a slender truss under first bending mode can have some members under translation mode. Care should be given according to the working scale.

Overall, equations (7) to (10) can be generalized as

$$
\begin{aligned}
& \delta W_{k, i}=\eta_{i}(t) \delta \eta_{i}(t) \\
& \oiiint_{\nu} \sum_{\nu} \Lambda_{\nu}(x, y, z)\left[\frac{\partial^{j} \varphi_{i}(x, y, z)}{\partial \nu^{j}}\right]^{2} d x d y d z
\end{aligned}
$$

where $\nu$ represents $x$-, $y$-, or $z$-direction; $\Lambda_{\nu}$ is the rigidity - translational (EA), flexural (EI), torsional (GJ), or shear $(\mathrm{G} \alpha \mathrm{A}) ; j=1$ if translation, torsional, or shear; and $j=2$ if flexion.

Substituting in the virtual displacement equality using equations (4) to (6) and equation (11), then rearranging and letting the equality be true for every $\delta \eta_{\mathrm{i}}(t)$

$$
\begin{aligned}
& \ddot{\eta}_{i}(t) \oiiint \varphi_{i}^{2}(x, y, z) r(x, y, z) \rho(x, y, z) d V+\dot{\eta}_{i}(t) \oiiint \varphi_{i}^{2}(x, y, z) c_{i}(x, y, z, t) d V \\
& +\eta_{i}(t) \oiiint \sum_{\nu} \Lambda_{\nu}(x, y, z)\left[\frac{\partial^{j} \varphi_{i}(x, y, z)}{\partial \nu^{j}}\right]^{2} d V=\oiiint_{i}(x, y, z, t) \varphi_{i}(x, y, z) d S
\end{aligned}
$$

Comparing with equation (1), one can identify, respectively, the equivalent modal mass, the equivalent modal damping, the equivalent modal stiffness, and the equivalent modal disturbance as

$$
\begin{gathered}
\tilde{m}_{i}=\oiiint \varphi_{i}^{2}(x, y, z) r(x, y, z) \rho(x, y, z) d V \\
\tilde{k}_{i}=\oiiint \sum_{\nu} \Lambda_{\nu}(x, y, z)\left[\frac{\partial^{j} \varphi_{i}(x, y, z)}{\partial \nu^{j}}\right]^{2} d V
\end{gathered}
$$

Now, equations (13a) and (13b) can be plugged into equation (2) to give for each mode $i$

$$
\frac{w_{d, i}^{2}}{1-\zeta_{i}^{2}}=\frac{\oiiint \sum_{\nu} \Lambda_{\nu}(x, y, z)\left[\frac{\partial^{j} \varphi_{i}(x, y, z)}{\partial \nu^{i}}\right]^{2} d V}{\oiiint\left(\varphi_{i}^{2}(x, y, z) r(x, y, z) \rho(x, y, z) d V\right.}
$$

or

$$
\begin{aligned}
& \oiint \sum_{\nu} \Lambda_{\nu}(x, y, z)\left[\frac{\partial^{j} \varphi_{i}(x, y, z)}{\partial \nu^{j}}\right]^{2} d V \\
& =\frac{w_{d, i}^{2}}{1-\zeta_{i}^{2}} \oiiint \varphi_{i}^{2}(x, y, z) r(x, y, z) \rho(x, y, z) d V
\end{aligned}
$$

\section{Proposed damage detection algorithm}

The proposed damage detection algorithm is buttressed in equation (14) using vibration signature. Indeed, sensors can be used on the structure in such a way that mode shapes, $\varphi_{i}$, modal frequencies, $w_{d, i}$, and modal damping ratios, $\zeta_{i}$, can be recorded during ambient vibrations $\left(p_{i}=0\right)$ or forced motions $\left(p_{i} \neq 0\right)$. This information can be obtained from vibrational signal using diverse methods such as fast Fourier transform combined with half-power band, curve-fitting method, or any other. In order to accurately record the vibration signature of structures, sensors should be adequately distributed throughout structures. Because recorded mode shapes are not continuous but discretized, equation (14) needs to be discretized as well.

\section{Discretization strategy}

Equation (14) can be described by equation (15) in a discretized domain, where $\Delta_{x}, \Delta_{y}$, and $\Delta_{z}$ are the tributary lengths to a grid point in $x$-, $y$-, and $z$-direction, respectively

$$
\begin{aligned}
& \sum_{x} \sum_{y} \sum_{z} \sum_{\nu} \Lambda_{\nu}(x, y, z)\left[\frac{\partial^{j} \varphi_{i}(x, y, z)}{\partial \nu^{j}}\right]^{2} \Delta_{z} \Delta_{y} \Delta_{x} \\
& =\frac{w_{d, i}^{2}}{1-\zeta_{i}^{2}} \sum_{x} \sum_{y} \sum_{z} \varphi_{i}^{2}(x, y, z) r(x, y, z) \rho(x, y, z) \Delta_{z} \Delta_{y} \Delta_{x}
\end{aligned}
$$

This equation involves either first derivative or second derivative of the mode shapes, which can be estimated using, for example, finite differences about $\nu$. Figure 2 illustrates the notations used in equation (15) in a structured grid for finite difference method (left) 


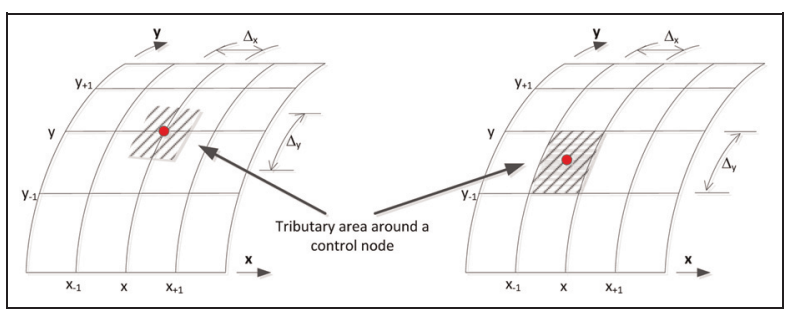

Figure 2. Structured grid system.

and for the finite volume method (right). Derivatives are presented for $\nu=x$ as example.

\section{Optimization as a solution strategy}

In practice, it is generally possible to record vibration signature for the first global modes. These modes can be qualified as translational, bending, torsional, or shearing by looking at mode shapes. Then, the corresponding forms of $\Lambda_{\nu}$ and the value of $j$ can be selected when writing equation (15) for each mode. This leads to a set of equations that are to be solved simultaneously for various fields that are mass, area, and moments of inertia such that the equivalent modal mass, $\tilde{m}_{i}$, and the equivalent modal stiffness, $\tilde{k}_{i}$, can be computed. It is important to note that, by assuming the structure remains elastic, Young's and shear moduli are kept fixed. The problem can be stated as demanding process is proposed later in the present article.

\section{Damage detection process}

Once the equivalent mass and stiffness arrays have been determined, two methods can be used to detect damages.

Method I: reference state. A reference state, set as undamaged state, can be used for comparison of its quantities, $\tilde{k}_{i}(x, y, z)$, and $\tilde{m}_{i}(x, y, z)$, versus those of a newly recorded state. Any deviation between two corresponding quantities, the one from an undamaged state and the one from the current state, can be interpreted as damage. This magnitude of the deviation can be considered as a measure of the damage severity and the corresponding node as the damage location. Particularly, a mass deviation may not be a damage event in a proper sense, but just a change in operational conditions. However, in the scope of this article, changes in operational condition will be assimilated as damage events for simplicity.

Method 2: scrutinize parameter continuity. In case there is no reference state, damage can be identified by scrutinizing the continuity of the parameter throughout the domain. Within a homogeneous subdomain, $\tilde{k}_{i}(x, y, z)$

Minimize $f\left(\Lambda_{\nu}, \rho\right)=\sum_{\text {mode } i}\left\{\frac{w_{d, i}^{2}}{1-\zeta_{i}^{2}}-\frac{\sum_{\text {domain }} \sum_{\nu} \Lambda_{\nu}(x, y, z)\left[\frac{\partial^{j} \varphi_{i}(x, y, z)}{\partial \nu^{\prime}}\right]^{2}}{\sum_{\text {domain }} \varphi_{i}^{2}(x, y, z) r(x, y, z) \rho(x, y, z) \Delta_{z} \Delta_{y} \Delta_{x}}\right\}^{2}$

Subject to $\Lambda_{\nu}(x, y, z) \geq 0$

and $\rho(x, y, z) \geq 0$

In order to solve for these various fields, Cauchy ${ }^{20}$ proposed a general method. In particular, depending on the problem configuration, Lagrange multiplier ${ }^{21}$ method can be used. Alternatively, gradient descent method, ${ }^{21}$ or any similar method, or any of their respective derivatives can be used in a space formed by $\Lambda$ and $\rho$ with the objective function defined by equation (16). The initial data for the variable fields can be chosen either as the nominal ones or as the values corresponding to a previous known state. If the process is at its first iteration, initial data can be taken as those estimated from structure geometry and material. If the process has been carried out in the past, initial data can be set either as in the previous case or as the results of previous attempt. A close-to-solution initial set of data will not only shorten the iterative procedure, but will also avoid possible local minima. A less computationally

and $\tilde{m}_{i}(x, y, z)$ are expected to show a continuity if no damage is present. Any abrupt change may denote an anomaly. On one hand, a peak or a valley in $\tilde{k}_{i}(x, y, z)$ may point out damage causing a sharp change of the mode shapes, their derivatives, or the rigidity fields. On the other hand, a peak or a valley in $\tilde{m}_{i}(x, y, z)$ may show a mass concentration or deconcentration.

\section{Discontinuity detection techniques}

Technique I: GSM. For a univariate response $f(x)$, a polynomial can be fitted at a moving point $x_{i}$ based on its neighboring points: $C\left(x_{i}\right)=a_{0}+a_{1} x_{i}+a_{2} x_{i}^{2}+a_{3} x_{i}^{3}=$ $\underline{g}_{i} \underline{\theta}_{i}^{T}$, with $\underline{g}_{i}=\left[1, x_{i}, x_{i}^{2}, x_{i}^{3}\right]$ and $\underline{\theta}_{i}=\left[a_{0}, a_{1}, a_{2}, a_{3}\right]$. The coefficients $a_{0}, a_{1}, a_{2}$, and $a_{3}$ are to be determined by least-squares estimation, for example. 
Technique 2: GFD. For a univariate response $f(x)$, Wang and $\mathrm{Qiao}^{10}$ proposed a generalized FD at the point $x$, center of the moving window $\left[x_{i}, x_{i+m}\right]$

$$
G F D_{m, s}(x)=\frac{\log (n)}{\log (n)+\log \left(d_{s} / L_{S}\right)}
$$

with

$$
\frac{L_{s}}{d_{s}}=\frac{\sum_{j=1}^{m} \sqrt{1+\left(1 / S^{2}\right)\left(\left(f\left(x_{i+j}\right)-f\left(x_{i+j-1}\right)\right) /\left(x_{i+j}-x_{i+j-1}\right)\right)^{2}}}{\max _{1 \leqslant j \leqslant m}\left(x_{i+j}-x_{i}\right) \sqrt{1+\left(1 / S^{2}\right)\left(\left(f\left(x_{i+j}\right)-f\left(x_{i+j-1}\right)\right) /\left(x_{i+j}-x_{i+j-1}\right)\right)^{2}}}
$$

where $m$ is the number of point intervals in the moving window; $n=L / \bar{\alpha}$ with $L$ as the domain length of $f(x)$ and $\bar{\alpha}$ as the average distance between successive points; $S$ is the scale parameter to be chosen sufficiently large (in this study, greater than $10^{3}$ ) to successfully detect damage. For the case of $S=1$, the technique reduces to the conventional FD. The GFD yields a large value at points where dissimilarities occur.

\section{Proposed damage detection strategy scheme}

Six main steps structure the workflow scheme of the proposed damage detection strategy, which gives pre-

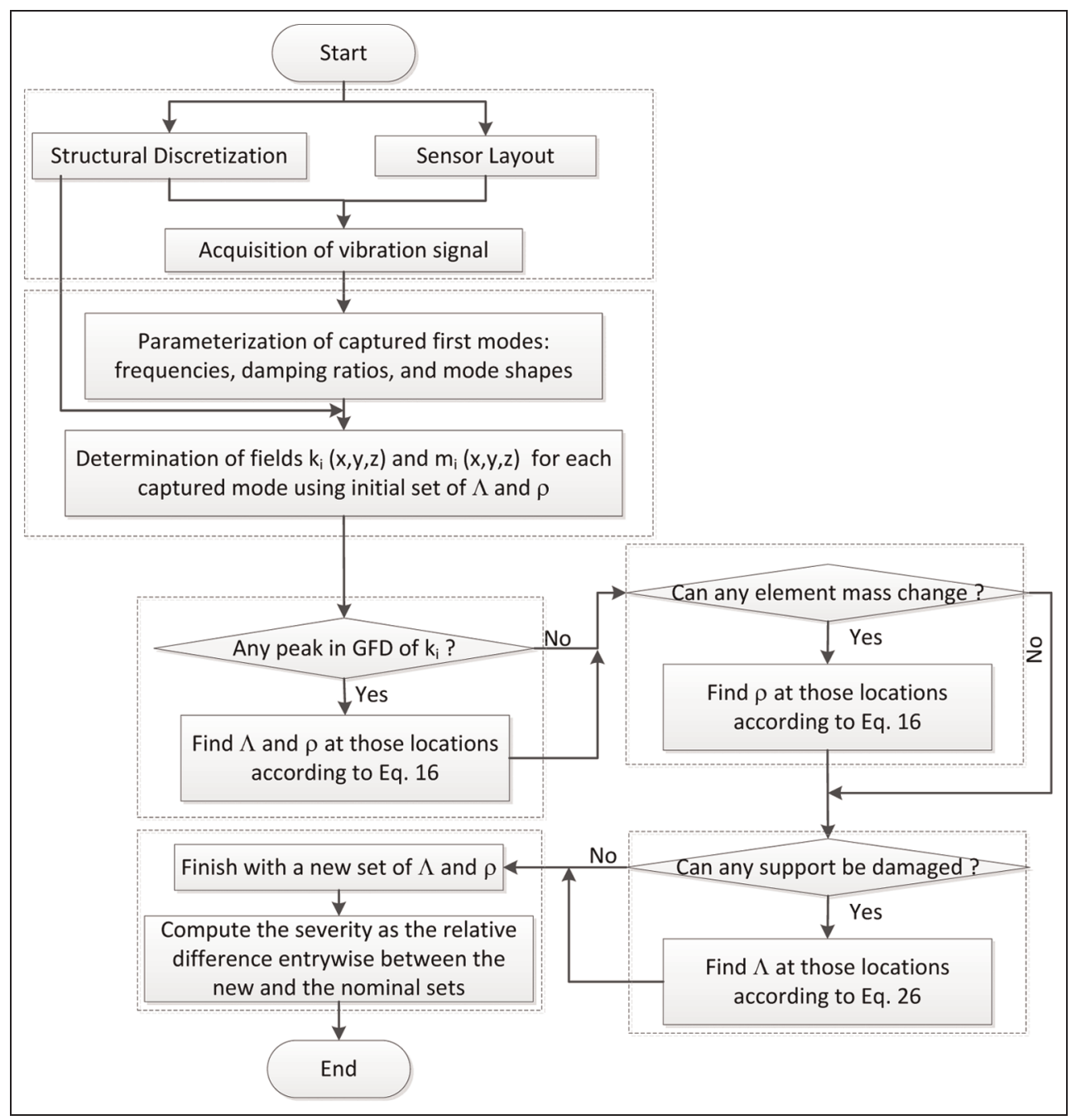

Figure 3. Flow chart for the proposed strategy. 
Table I. Description of the case studies.

\begin{tabular}{llll}
\hline Material properties & Concrete & Aluminum \\
\hline Mass density, $\rho\left(\mathrm{kg} / \mathrm{m}^{3}\right)$ & 2500 & 2700 & 7850 \\
Young's modulus, $E(\mathrm{GPa})$ & 25 & 69 & 200 \\
Specified strength, $f_{c} /$ yield stress, $f_{y}(\mathrm{MPa})$ & 25 & 95 & 350 \\
Poisson's ratio, $\nu$ & 0.2 & 0.35 & 0.3 \\
\hline
\end{tabular}

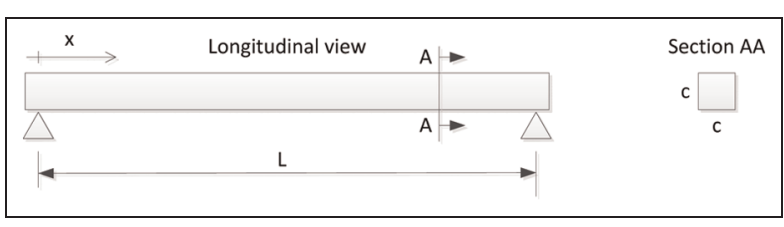

Figure 4. Case study I: simply supported concrete beam.

the choice of a grid system and to the initialization of the rigidity fields and the fields of mass densities. Concurrently, an optimal arrangement of the sensors throughout the structure will be determined keeping in mind that critical structural locations should be specially considered. It is obvious that refinement of the mesh size will increase accuracy and resolution when locating potential damages. Care should be also taken at the changes in geometry and material.

The solution domain of equation (16) can be restricted to the subdomain where discontinuity has been observed, setting the values of the design parameters being equal to their nominal values outside of the damaged subdomain. This strategy fastens the solution process while avoiding inoperative solution points. The damage severity can then be obtained as the relative deviation between the design parameter values obtained from equation (16) and their nominal values.

\section{Numerical case studies}

\section{Description of case studies}

In order to show the efficiency of the proposed algorithm, three numerical case studies are selected. They concern a concrete beam, an aluminum plate, and a steel monopod offshore platform. Table 1 describes the principal characteristics of each material for the case studies.

The concrete beam is a $c=0.2-\mathrm{m}$ side square section extruded along an $L=5$-m-long straight axis and rests on two pin supports, as shown in Figure 4. Figure 5 depicts the aluminum plate, object of second case study. It is a $0.5-\mathrm{m}$-wide and $0.8-\mathrm{m}$-long plate and has a thickness of $0.002 \mathrm{~m}$. All its borders are clamped. The steel platform is adapted from Elshafey et al. ${ }^{22}$ It is

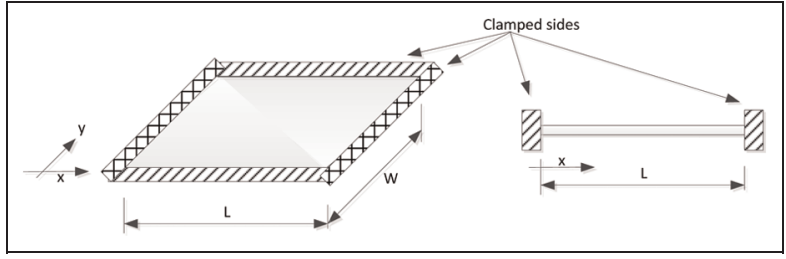

Figure 5. Case study 2: clamped edge aluminum plate.

made of two cylindrical sections with different diameters connected by a frustoconical part. It supports a deck whose nominal total mass is $3000 \mathrm{~kg}$, which can vary within a $10 \%$ interval. As illustrated by Figure 6 , the foundation, located at $H w=25.50 \mathrm{~m}$ beneath the mean sea level, is assumed fixed (all degrees of freedom are restrained) in normal conditions. The monopod dimensions are $O D_{1}=210 \mathrm{~cm}, W T_{1}=5 \mathrm{~cm}$, $O D_{2}=150 \mathrm{~cm}, \quad W T_{2}=4 \mathrm{~cm}, \quad H_{1}=25.00 \mathrm{~m}$, $H_{2}=20.00 \mathrm{~m}$, and $H_{3}=1.00 \mathrm{~m}$. The wall thickness of the frustoconical part varies from $5 \mathrm{~cm}$ at the bottom to $4 \mathrm{~cm}$ at the top.

For each of these structures, a numerical model is built up using the commercial software package SAP2000 version 15.1.0. Several damages are intentionally placed at different locations of each case study. On each structure, three scenarios are defined with damage variation in terms of number, nature, position, and severity. Especially in case study 2, all damages are cracks through the full plate thickness oriented either along $y$-direction $(d y)$ or $x$-direction $(d x)$. In general, damages are simulated by decreasing section modifiers (area and inertia accordingly) at their locations by a certain percentage that will be referred later as damage severity. For the crack at the support in case study 2 , section modifiers have been set to zero. In case study 3 , the foundation damage is simulated by the loss of stiffness of the bottommost element. Table 2 summarizes all the damage scenarios assigned to each case study, respectively.

\section{Results from the numerical simulations}

From case study 1, GSM applied on curvature, GFD technique applied on curvature, GFD technique applied 
Table 2. Damage scenarios.

\begin{tabular}{|c|c|c|c|c|}
\hline & ID & Location & Severity & Observations \\
\hline \multicolumn{5}{|l|}{ Case study I } \\
\hline Damage I & IDI & $x=2 \mathrm{~m}$ & $10 \%$ & One damage \\
\hline Damage 2 & ID2 & $\begin{array}{l}x=2 \mathrm{~m} \\
x=4.5 \mathrm{~m}\end{array}$ & $\begin{array}{l}20 \% \\
5 \%\end{array}$ & Two damages simultaneously \\
\hline Damage 3 & ID3 & $\begin{array}{l}x=2 \mathrm{~m} \\
x=4.5 \mathrm{~m} \\
x=3.5 \mathrm{~m}\end{array}$ & $\begin{array}{l}70 \% \\
15 \% \\
15 \%\end{array}$ & Three damages simultaneously \\
\hline \multicolumn{5}{|l|}{ Case study 2} \\
\hline Damage I & 2DI & $\begin{array}{l}x=0.2 \mathrm{~m} \\
y=0.2 \mathrm{~m}\end{array}$ & $d y=0.05 \mathrm{~m}$ & Full opening \\
\hline Damage 2 & 2D2 & $\begin{array}{l}x=0.2 \mathrm{~m} \\
y=0.2 \mathrm{~m} \\
x=0.6 \mathrm{~m} \\
y=0.4 \mathrm{~m}\end{array}$ & $\begin{array}{l}d y=0.05 \mathrm{~m} \\
d y=0.10 \mathrm{~m}\end{array}$ & Two full openings simultaneously \\
\hline Damage 3 & 2D3 & $\begin{array}{l}x=0: 0.8 \mathrm{~m} \\
y=0 \mathrm{~m}\end{array}$ & $d x=0.80 \mathrm{~m}$ & Crack at support \\
\hline \multicolumn{5}{|l|}{ Case study 3} \\
\hline Damage I & 3DI & $z=26 m$ & $10 \%$ & One damage \\
\hline Damage 2 & 3D2 & $z=46 m$ & $7.5 \%$ & Change in operational condition (deck mass) \\
\hline Damage 3 & 3D3 & $z=0 m$ & $25 \%$ & Foundation damage \\
\hline
\end{tabular}

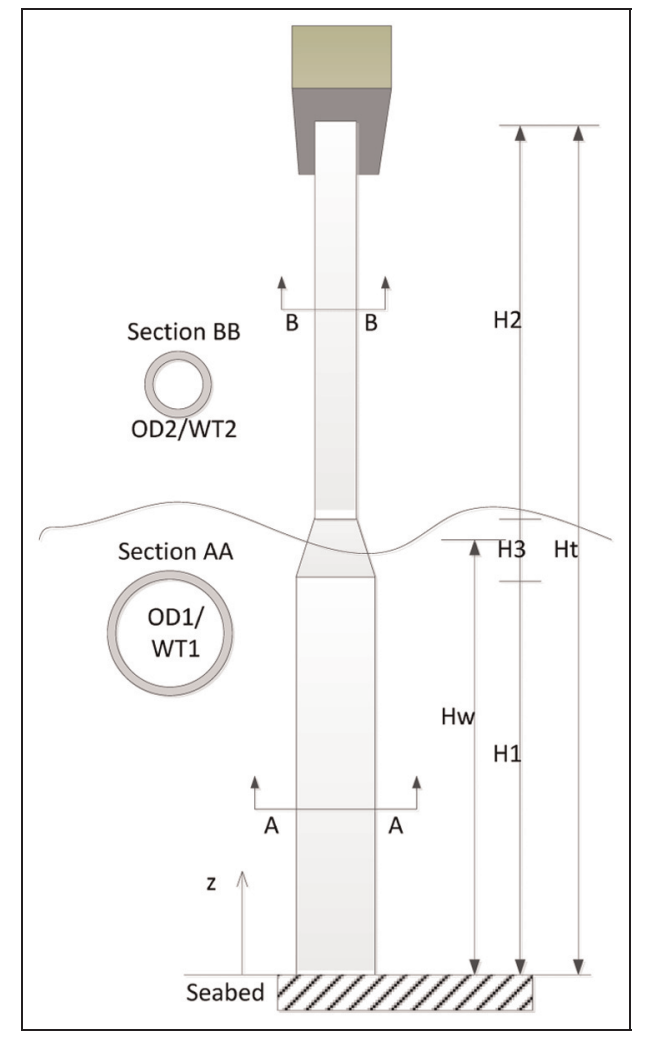

Figure 6. Case study 3: steel monopod platform.

on $\tilde{k}$, and damage severity as described in this article have been computed and are depicted in Figure 7. GSM and GFD effectively spot the damages, and the damage severity estimates the actual severity of the damage. Figure 8 presents the detected location of damages on case study 2 based on GFD applied on $\tilde{k}$. Here, damage severities have not been predicted as it is assumed that a crack exists only if its severity is $100 \%$. From case study 3 , only GFD applied on $\tilde{k}$ and damage severities are presented in Figure 9. Table 3 summarizes all the results on the numerical cases.

\section{Experimental case study}

\section{Description of the case study and the experimental setup}

In addition to the numerical cases, one experimental case was studied in laboratory. The structure consists of a $1 \mathrm{~cm} \times 1 \mathrm{~cm}$ section metallic beam resting on three simple supports as illustrated in Figure 10. The two spans are, respectively, of $88.0 \mathrm{~cm}$ and $46.0 \mathrm{~cm}$. A $60.0 \%$ severity damage is purposefully created by a saw cut at $x=46.5 \mathrm{~cm}$ from the end of the long span (considered as the origin of the abscissa). The setup is equipped with a linear variable differential transformer (LVDT) and strain gauges as shown in Figure 10. The information collected from the strain gauges is not of the prime importance for this study, but has only served as cross-check. The experiment consists of recording at the LVDT location $(x=55 \mathrm{~cm})$ the vertical displacement created by a $5-\mathrm{kg}$ mass successively placed at 14 different locations along the damaged bar. This corresponds to the construction of influence lines relative to the LVDT location for a vertical force as presented in Figure 11. 
Table 3. Summary of the results of the numerical cases.

\begin{tabular}{ccccc}
\hline & ID & Actual severity & Detected severity & Error = (Actual - Detected $)$ \\
\hline Case study I & & & & $-5.00 \%$ \\
Damage I & IDI & $10.00 \%$ & $15.00 \%$ & $-5.50 \%$ \\
Damage 2 & ID2 & $20.00 \%$ & $25.50 \%$ & $-2.50 \%$ \\
& & $5.00 \%$ & $7.50 \%$ & $-1.00 \%$ \\
Damage 3 & ID3 & $70.00 \%$ & $71.00 \%$ & $+13.00 \%$ \\
& & $15.00 \%$ & $7.00 \%$ & $+8.00 \%$ \\
Case study 3 & $15.00 \%$ & $10.10 \%$ & $-0.10 \%$ \\
Damage 1 & 3DI & $10.00 \%$ & $6.50 \%$ & $+1.00 \%$ \\
Damage 2 & 3D2 & $7.50 \%$ & $20.79 \%$ & $+4.21 \%$ \\
Damage 3 & 3D3 & $25.00 \%$ & & \\
\hline
\end{tabular}

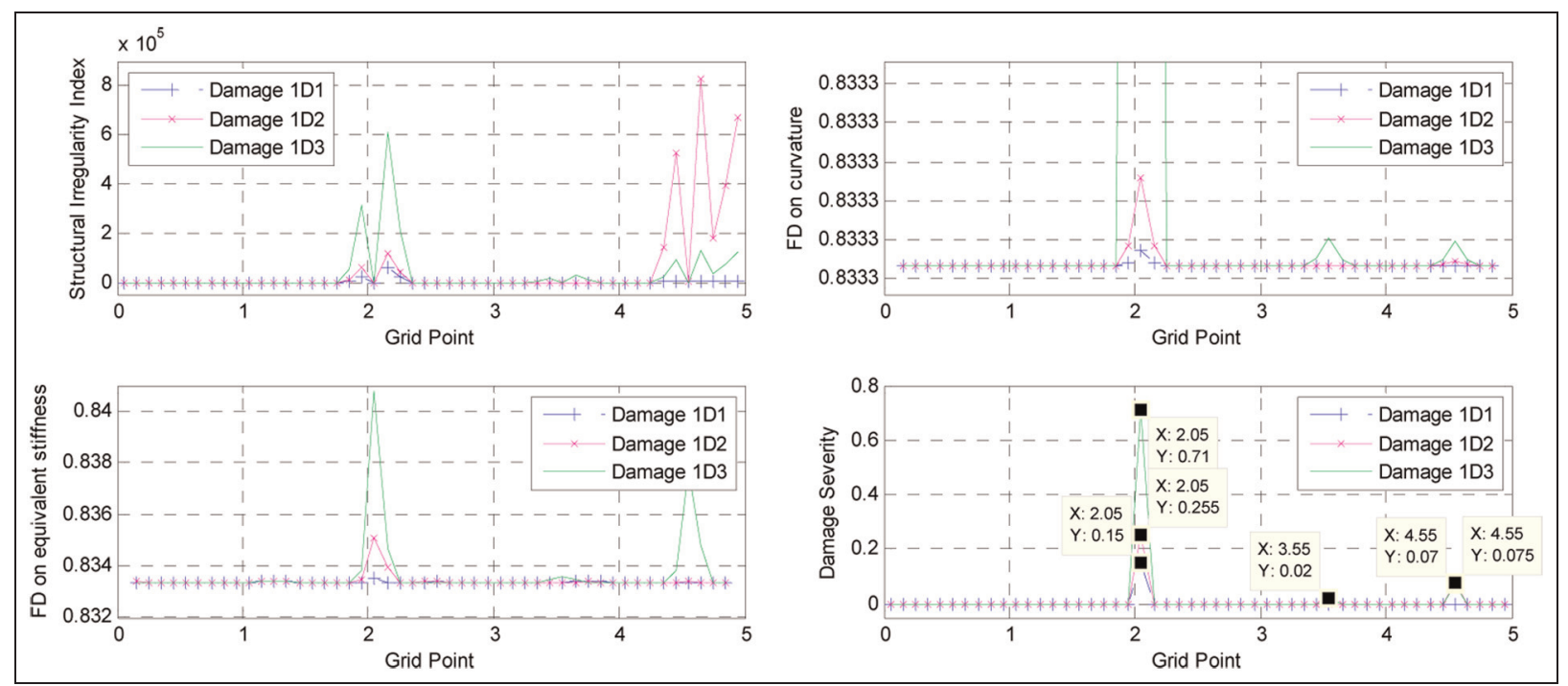

Figure 7. Case study I: GSM on curvature (top-left), GFD on curvature (top-right), GFD on $\tilde{k}$ (bottom-left), and damage severity (bottom-right).

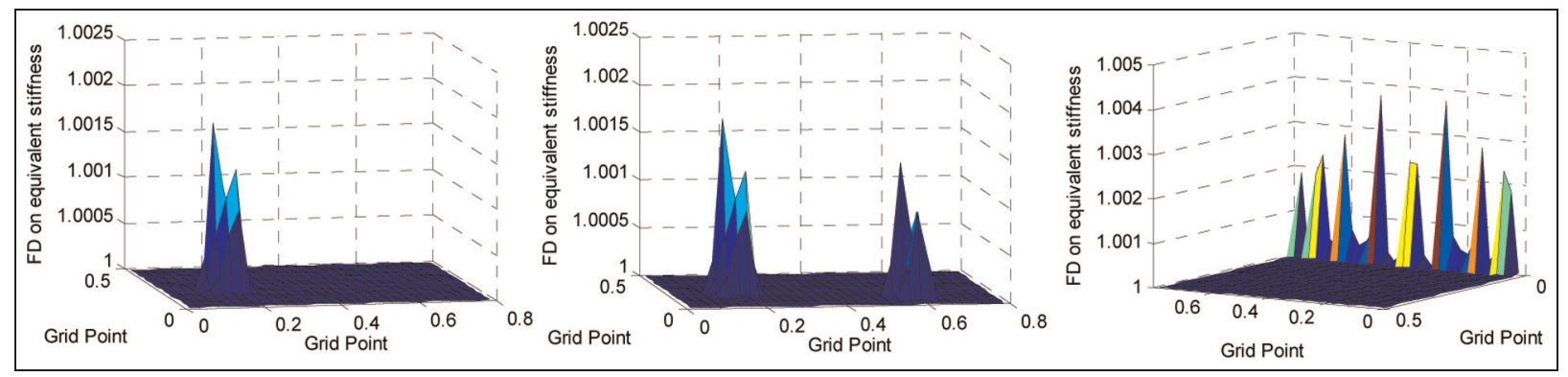

Figure 8. Case study 2: GFD on $\tilde{k}$.

A digital model is prepared in the platform SAP2000 with the aim of mimicking the laboratory model. The gross geometry and the boundary conditions are depicted by Figure 10. The crack is modeled as partial loss of structural performance of the corresponding beam portion: area and section inertia are modified.
The material properties and the crack parameters have been set such that the influence line from the digital model matches that of the experimental test. The updating process was carried out by trial-and-error method. Table 4 summarizes the initial/updated values of the design variables. 

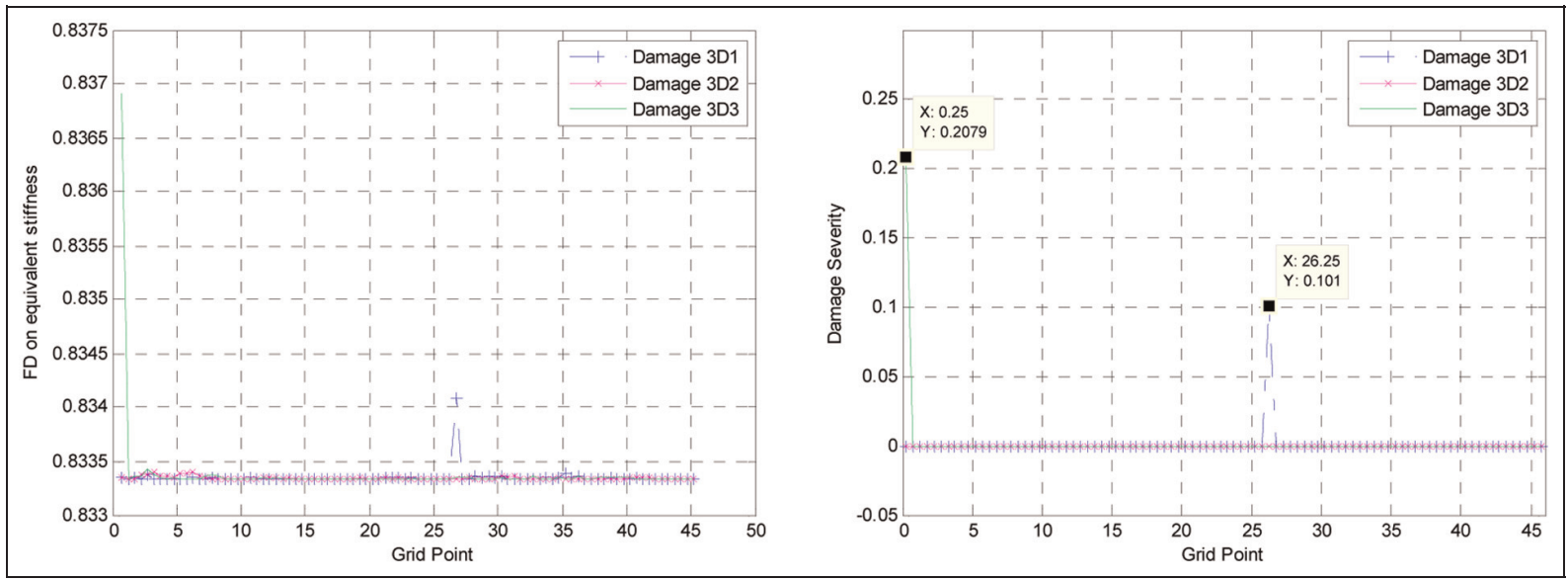

Figure 9. Case study 3: GFD on $\tilde{k}$ and damage severity.

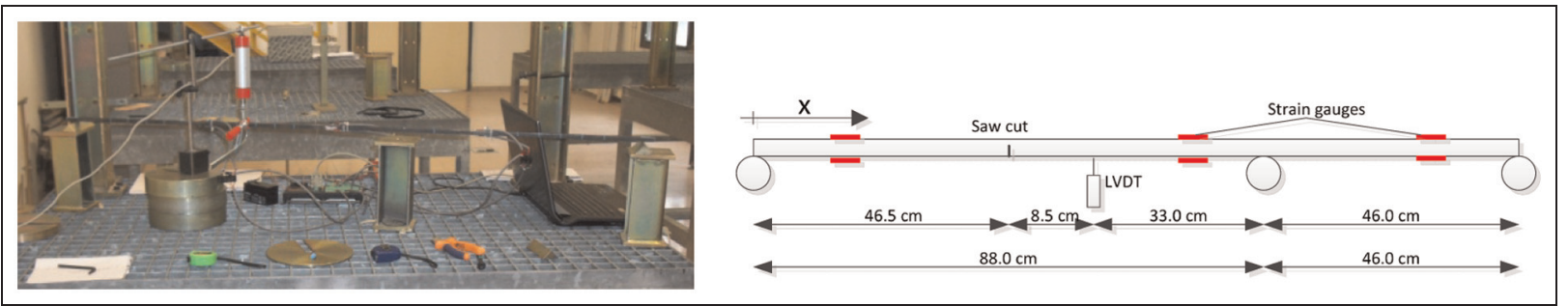

Figure 10. Experiment setup: photograph and sketch.

Table 4. Design variables for the structural identification.

\begin{tabular}{llll}
\hline & Unit & Initial value & Updated value \\
\hline $\begin{array}{l}\text { Material properties } \\
\text { Elastic modulus of }\end{array}$ & $\mathrm{GPa}$ & 93 & 90 \\
$\begin{array}{l}\text { the gray cast iron } \\
\begin{array}{l}\text { Crack parameters } \\
\text { Location }\end{array}\end{array}$ & $\mathrm{cm}$ & 46.5 & \\
$\begin{array}{l}\text { Opening } \\
\text { Area modifier }\end{array}$ & $\mathrm{mm}$ & 1.5 & 45.1 \\
Inertia modifier & - & $10^{-1}$ & 2.2 \\
\hline
\end{tabular}

A modal analysis is conducted on the calibrated digital model, from which the modal properties are extracted. As for the numerical cases, the process is repeated for the digital model.

\section{Results from the experimental test}

As in case study 3, Figure 12 depicts the results of GFD applied on $\tilde{k}$ and damage severity. It shows an estimated damage severity of $71.5 \%$, which is an error of $-11.5 \%$ with respect to the actual damage severity.

\section{Discussion}

The observation of the results obtained in this study reveals several trends. From the first case study, GSM

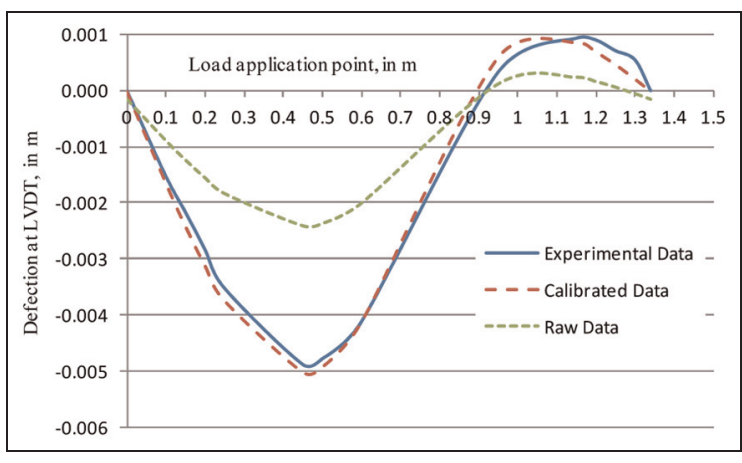

Figure I I. Structural identification results.

has detected only damaged edges as also found by Yoon et al. ${ }^{7}$ that makes the results difficult for interpretation (see Figure 7). More precisely, the GFD technique points out the damage location accurately. Applied on $\tilde{k}$, the GFD technique reveals to be better in detecting damage compared to the same technique applied on the curvature. Indeed, Figure 7 shows that the FD for the former case ranges from 0.833 to 0.841 , while for the latter case, the range of variation is very narrow around the value of 0.833 , requiring a finer resolution for interpretation. Nevertheless, the GFD technique applied on curvature seems to keep the proportions of each damage severity. 


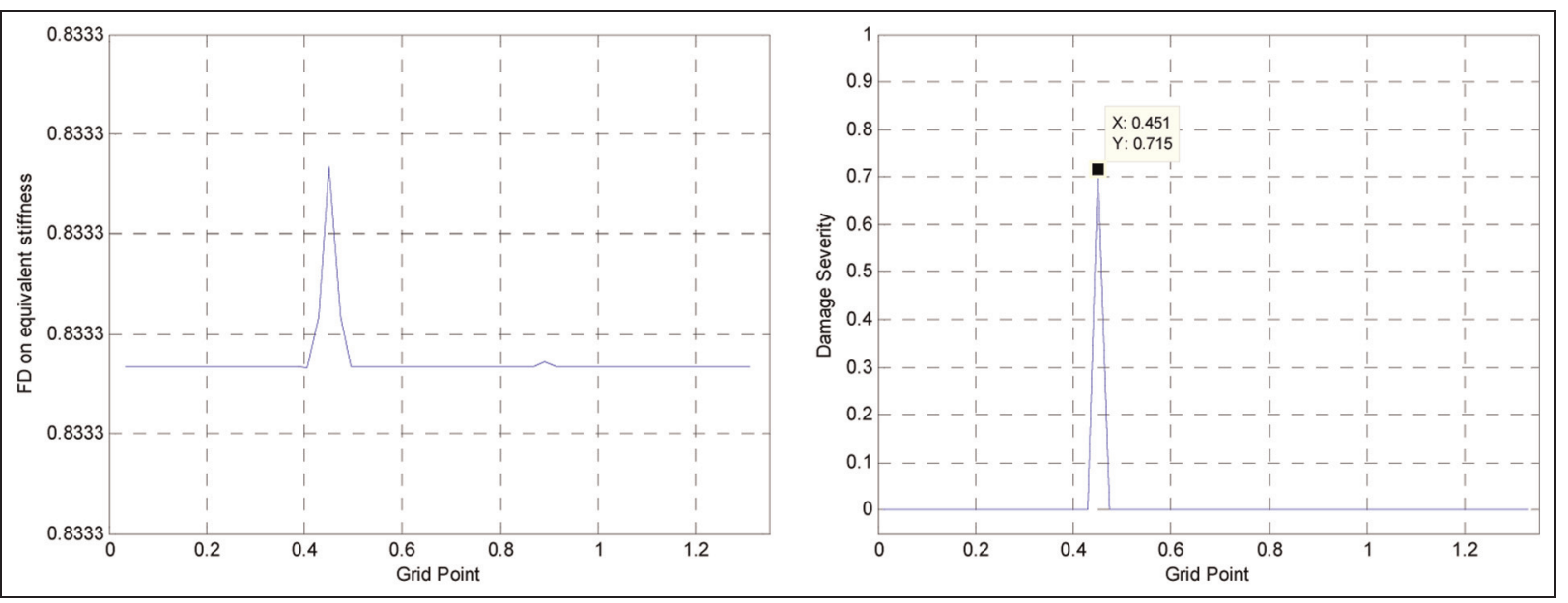

Figure I2. Experimental case study: GFD applied on $\tilde{k}$ and damage severity.

In the presently proposed methodology, estimating damage severity is a step separated from detecting damages. For ensuring a better damage detection, the GFD technique applied on $\tilde{k}$ was used for the rest of the study. The setup was able to successfully detect all damages in terms of existence and location. However, it should he kept in mind that location accuracy is limited due to the mesh resolution.

Damage severities are estimated with an appropriate accuracy except for scenario 1D3 where we have three simultaneous damages. The relatively poor performance in quantifying the least significant damages is due to the fact that the most severe damage has introduced nonlinearity in the structure. This violates the hypothesis set when deriving this method. However, since damages rarely occur with high severity at their apparition, this does not constitute a drawback for the procedure.

The GFD technique as carried out for case study 2 was conducted along the $x$-dimension of the plate. It did not only identify damage at the actual location but also in adjacent locations. The reason is because the GFD technique, as defined in Wang and Qiao, ${ }^{10}$ is developed for a 1D structure. Therefore, further investigations may update the method in order to have it more suitable for $2 \mathrm{D}$ structures. Besides, the support crack was also well detected but represented by a highly oscillating FD. This is due to the averaging effect of the FD computed on $\tilde{k}$ for the first five modes $(i=1-5)$.

In case study 3 , it was seen that the proposed technique is able to detect and differentiate between a crack, a change in mass, and a foundation problem. Therefore, an additional use of this tool can be the measure of the actual mass on a platform deck and to check whether it is between the prescribed bounds. In detecting damage severity, the chosen solution procedure (Figure 3) demonstrates its ability to search optimal points in equation (16) without altering intact location parameters; the parameters at non-damaged locations could be altered if a general search algorithm had been used.

In the experimental case, the damage was also localized satisfactorily by the GFD technique applied on $\tilde{k}$. Some sensitivity to the middle support properties was observed. The $-11.5 \%$ error in severity is caused by the cumulative errors coming from structural identification, measurements, numerical, and computational operations. With a direct measurement of the vibrational properties, the deviation in severity estimation would have been smaller than the one obtained here, because there would not be any structural identification or computational operations.

Despite these satisfactory results, the major drawback of this method is that it relies on numerical derivation followed by integration. To achieve sufficient accuracy, these operations require a good discretization, which, for its implementation, may need numerous sensors or sophisticated ones like laser-based system, ${ }^{23}$ roved sensors, ${ }^{13}$ or (thermal) imaging techniques.

\section{Conclusion}

This article presents a novel method of damage detection, which measures actual damage severity and changes in mass properties, without using baseline structure or the mathematical model of the system. The method is based on Rayleigh's quotient derived in three dimensions by employing the virtual energy principle. Theoretical developments have been considered to build up this method. Some computational techniques are proposed and their efficacies are discussed. Field implementation strategies are also suggested in order to facilitate the online application of the method. One experimental case and three numerical case studies were 
implemented to assess the efficiency of the proposed technique. The results revealed its ability to successfully spot damages and to estimate their respective severities with adequate accuracy in general without any false damage identification, thanks to the intrinsic attributes of the mathematical procedures used. It has been shown that the proposed strategy encompasses the features coming from frequency shift, damping, mode shapes and their derivatives, and FD to achieve its goal. Overall, the main power of this technique is that it does not require any dynamic measurements coming from a baseline structure but succeeds in determining physically meaningful parameters. Thus, it is a good tool for structures of any age: old or newly constructed, and any regularity of damage detection campaign: periodically or continuously. It has been seen, however, that upgrading the GFD technique from $1 \mathrm{D}$ to $2 \mathrm{D}$ is a topic which may need future research attention.

\section{Acknowledgements}

The author would like to express his gratitude to Prof. Ahmet Türer, who collaborated with him during his stay at the Middle East Technical University, Ankara, Turkey, where this study has been initiated. The academic resources (laboratory facilities and software packages) found there were of great support.

\section{Declaration of conflicting interests}

The author(s) declared no potential conflicts of interest with respect to the research, authorship, and/or publication of this article.

\section{Funding}

The author(s) received no financial support for the research, authorship, and/or publication of this article.

\section{References}

1. Carden EP and Fanning P. Vibration based condition monitoring: a review. Struct Health Monit 2004; 3(4): 355-377.

2. Abdo MAB and Hori MA. Numerical study of structural damage detection using changes in the rotation of mode shapes. J Sound Vib 2002; 251(2): 227-239.

3. Yan YJ, Yam LH, Cheng L, et al. Modeling method of damage structures for structural damage detection. Compos Struct 2006; 72: 193-199.

4. Gawronski W and Sawicki JT. Structural damage detection using modal norms. J Sound Vib 2000; 229(1): 194-198.

5. Elshafey AA, Haddara MR and Marzouk H. Damage detection in offshore structures using neural networks. Mar Struct 2010; 23: 131-145.
6. Salehi M, Ziaei-Rad S, Ghayour M, et al. Structural damage detection technique based on measured frequency response functions. Contemp Eng Sci 2010; 3(5): 215-226.

7. Yoon MK, Heider D, Gillespie JW Jr, et al. Local damage detection using the two-dimensional gapped smoothing method. J Sound Vib 2005; 279: 119-139.

8. Li $\mathbf{J}$ and Hao H. Substructure damage identification based on wavelet-domain response reconstruction. Struct Health Monit 2014; 13(4): 389-405.

9. Lee SG, Yun GJ and Shang S. Reference-free damage detection for truss bridge structures by continuous relative wavelet entropy method. Struct Health Monit 2014; 13(3): 307-320.

10. Wang $\mathbf{J}$ and Qiao P. Improved damage detection for beam-type structures using a uniform load surface. Struct Health Monit 2007; 6(2): 99-110.

11. Viero PF and Roitman N. Application of some damage identification methods in offshore platforms. Mar Struct 1999; 12: 107-126.

12. Ruotolo R, Surace C and Worden K. Application of two damage detection techniques to an offshore platform. Shock Vib Digest 2000; 32: 30-31.

13. Nguyen T, Chan THT and Thambiratman DP. Effects of wireless sensor network uncertainties on output-only modal analysis employing merged data of multiple tests. Adv Struct Eng 2014; 17(3): 319-329.

14. Reynders E, Wursten G and de Roeck G. Output-only structural health monitoring in changing environmental conditions by means of nonlinear system identification. Struct Health Monit 2014; 13(1): 82-93.

15. Ubertini F, Comanducci G and Cavalagli N. Vibrationbased structural health monitoring of a historic bell-tower using output-only measurements and multivariate statistical analysis. Struct Health Monit 2016; 15(4): 438-457.

16. Comanducci $G$, Magalhães $F$, Ubertini $F$, et al. On vibration-based damage detection by multivariate statistical techniques: application to a long-span arch bridge. Struct Health Monit 2016; 15(5): 505-524.

17. Chopra AK. Dynamics of structures: theory and applications to earthquake engineering. Englewood Cliffs, NJ: Prentice Hall, 1995, p. 729.

18. Wilson JF. Dynamics of offshore structures. Hoboken, NJ: John Wiley \& Sons, 2003, p. 325.

19. Karamchandani KC, Gupta NK and Pattabiraman J. Evaluation of percent critical damping of process towers. Hydrocarb Process 1982; 61: 205-208.

20. Cauchy A. Méthode générale pour la résolution des systèmes d'équations simultanées. Comp Rend Hebd Séance Acad Sci 1847; 25: 536-538.

21. Arfken GB, Weber HJ and Harris FE. Mathematical methods for physicists. 7th ed. Oxford: Elsevier, 2013, p. 1179 .

22. Elshafey AA, Haddara MR and Marzouk H. Identification of excitation and reaction forces spectra for offshore platforms. Can J Civ Eng 2010; 37: 66-76.

23. Chen S-E. Laser scanning technology for bridge monitoring. In: Rodriguez JAM (ed.) Laser scanner technology. Rijeka: InTech, 2012, pp. 71-92. 